\title{
Two-Year Comparative Outcomes of First- and Second-Generation Trabecular Micro-Bypass Stents with Cataract Surgery
}

\author{
Ricardo Augusto Paletta \\ Guedes' \\ Daniela Marcelo Gravina' \\ Vanessa Maria Paletta \\ Guedes' \\ Alfredo Chaoubah ${ }^{2}$ \\ 'Glaucoma Department, Paletta Guedes \\ Eye Institute, Juiz de Fora, MG, Brazil; \\ ${ }^{2}$ Statistics Department, Federal \\ University of Juiz De Fora, Juiz de Fora, \\ MG, Brazil
}

\begin{abstract}
Introduction: This retrospective comparative study assessed real-world effectiveness and

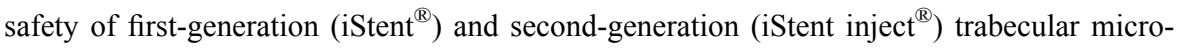
bypass stents with cataract surgery in patients with open-angle glaucoma (OAG).
\end{abstract}

Material and Methods: Through a 24-month postoperative follow-up, the effectiveness was quantified by intraocular pressure (IOP) reduction; mean glaucoma medication reduction; proportional analysis of eyes meeting IOP cutoffs $(<18,<15,<12 \mathrm{mmHg})$ either with or without medications; and proportional analysis of medication burden. Safety measures included visual acuity, adverse events, and secondary surgery.

Results: A total of 82 consecutive eyes (39 iStent, 43 iStent inject) with a 24-month followup were analyzed. Most eyes $(74.4 \%)$ had primary open-angle glaucoma, with the remainder having pseudoexfoliative or pigmentary glaucoma; all eyes had mild-to-moderate disease. At 24 months postoperative, the mean IOP was lower, and the percent reduction from baseline was greater, in iStent inject eyes $(26.0 \%$ reduction, $17.7 \mathrm{mmHg}$ to $13.1 \mathrm{mmHg})$ than in iStent eyes $(9.8 \%$ reduction, $16.4 \mathrm{mmHg}$ to $14.8 \mathrm{mmHg}$ ) (between-groups comparison, $\mathrm{p}=0.019$ ). Within each group, the postoperative IOP reduction was greater in eyes with higher baseline IOP $(\mathrm{p}<0.001)$. Medication burden decreased significantly in both groups, from 1.74 to 0.51 mean medications for iStent $(70.7 \%$ reduction, $\mathrm{p}<0.0001)$, and 2.19 to 0.65 for iStent inject ( $70.3 \%$ reduction, $\mathrm{p}<0.0001)$. Both groups exhibited excellent safety.

Conclusion: iStent or iStent inject with phacoemulsification produced significant IOP and medication reductions, with effects enduring for two years. IOP reductions were greater for iStent inject than for iStent. Within each group, higher preoperative IOP was associated with greater postoperative IOP reduction.

Keywords: iStent, iStent inject, trabecular micro-bypass, micro-invasive, MIGS

\section{Introduction}

Over the past decade, there has been a considerable expansion in the number of medical and surgical modalities available to treat glaucoma, the top cause of irreversible blindness around the world. ${ }^{1}$ Treatments can range from topical medications (conservative) to filtration surgeries such as trabeculectomy and tube-shunt implantation (more invasive). As an intermediate intervention along this treatment continuum, an increasingly utilized option is micro-invasive glaucoma surgery (MIGS). Although MIGS procedures typically do not achieve the same dramatic intraocular pressure (IOP) reduction as traditional filtration surgeries, they do not carry the same risk of sight-threatening complications (eg, endophthalmitis,
Correspondence: Ricardo Augusto Paletta Guedes

Glaucoma Department, Paletta Guedes Eye Institute, 79, Oscar Vidal streeet, Juiz de Fora, MG, 360I0-060, Brazil

Tel: $+55323213-1927$

Email palettaguedes@yahoo.com 
hypotony, choroidal detachment or effusion, bleb-related infections, etc.). ${ }^{2-4}$ In the large segment of patients whose disease does not yet warrant the risk of filtration surgery, MIGS procedures can offer a lower-risk, adequately efficacious treatment option.

The first United States Food and Drug Administration (FDA)-approved MIGS device, the iStent, as well as the second-generation iStent inject, both act upon the natural physiologic trabecular outflow pathway in patients with open-angle glaucoma (OAG). By creating a patent conduit between the anterior chamber and Schlemm's canal (and thereby to the episcleral venous plexus), the stents are designed to bypass the diseased trabecular meshwork into which they are placed. The iStent and iStent inject stents are composed of the same biocompatible, implant-grade titanium, and share the same mechanism of action; however, a number of key attributes distinguish the two devices. The most salient differences include the presence of two (iStent inject) versus one (iStent) stent, to enable access to more collector channels; four outlet lumens (iStent inject) versus one (iStent), for multidirectional aqueous outflow; and a straightforward (iStent inject) versus angled (iStent) surgical approach, for easier implantation. A substantial body of evidence exists for both devices, including studies in patients with various glaucoma subtypes, severities, and ethnicities; in standalone usage or combined with phacoemulsification; in realworld and FDA-trial settings; and in ancillary areas of research such as cost-effectiveness, ocular surface disease, refractive neutrality, and patient quality of life. ${ }^{5-40}$

Three prior publications, including two from the authors ${ }^{38,39}$ and one by Manning in Australia, ${ }^{40}$ compared outcomes of either device with cataract surgery in their respective patient populations. Results across the studies showed favourable safety and efficacy of both devices, while consistently demonstrating greater benefits for iStent inject over iStent in terms of mean IOP reduction, attainment of prespecified IOP endpoints, mean medication burden, medication reduction from baseline, and percent of patients becoming medication-free. The studies also showed highly favourable safety outcomes which have come to characterize both devices.

The purpose of the present study is to evaluate the safety and effectiveness of iStent and iStent inject in a larger and longer-term (two-year) cohort of OAG patients within the author's real-world patient population. This report constitutes the first two-year comparative analysis of the two devices to-date, providing information with direct clinical relevance to practicing surgeons and their patients.

\section{Materials and Methods Study Design and Patient Population}

In this longitudinal retrospective study, we evaluated all consecutive eyes that underwent implantation of either iStent or iStent inject combined with cataract surgery. All surgeries were performed in a single centre by one surgeon (R.G.). Choice between implants was largely based on the availability of the different implants on the Brazilian market. From June 2017 to November 2017, only first-generation trabecular micro-bypass was available for use. After the secondgeneration implant was approved for use (November 2017), the majority of cases migrated to it. In infrequent cases, patients' ability to pay for either device influenced implant selection. No clinical variable, such as level of IOP or glaucoma stage, drove the indications for either implant.

All patient charts were assessed for potential inclusion in the cohort using the following inclusion criteria: iStent or iStent inject surgery with phacoemulsification cataract surgery; age $>18$ years old; mild to moderate OAG [mean deviation (MD) no worse than $-12 \mathrm{~dB}$ in Humphrey Visual Field Test]; current treatment with 0 to 4 glaucoma medications; surgery from June 2017 to June 2018 (ie, having at least 24 months of follow-up); and no significant ocular comorbidities that would confound glaucoma parameters. Exclusion criteria included follow-up less than 24 months; chronic or acute intraocular inflammation; angle closure; and the surgeon's first 10 iStent cases and first 5 iStent inject cases (to minimize any potential learningcurve bias). The study was performed in accordance with the ethical standards of the Declaration of Helsinki (as revised in Brazil 2013), including informed consent of patients; it was approved by the Ethics Committee of the Santa Casa de Misericordia de Juiz de Fora.

\section{Outcome Measures and Statistical Analysis}

In addition to the demographic data of patients (age, sex, race, type of glaucoma, glaucoma stage), we evaluated the change in IOP, number of glaucoma medications, and visual acuity from the preoperative period to the end of the follow-up. Effectiveness outcomes included mean IOP reduction (\%); reduction in mean number of medications (\%); rates of "qualified success" (with adjunctive glaucoma medications as needed) at different IOP levels (IOP $<18 \mathrm{mmHg},<15$ 
$\mathrm{mmHg},<12 \mathrm{mmHg}$ ); rates of "unqualified success" (with no adjunctive glaucoma medications) at different IOP levels (IOP < $18 \mathrm{mmHg},<15 \mathrm{mmHg},<12 \mathrm{mmHg}$ ); and survival analysis of the probability of qualified and unqualified success at IOP $<18 \mathrm{mmHg}$ through 2 years postoperative. Safety outcomes included visual acuity; intra- and post-operative adverse events; and secondary glaucoma interventions.

All numerical variables were tested for normality. For variables with normal distribution, a Student's $t$-test was used for comparisons. For non-normal distribution, a nonparametric test was used (Kruskal-Wallis or Mann-Whitney test). Chi-square testing was used for the analysis of categorical variables. A p-value of $<0.05$ was considered statistically significant. All statistical analyses were performed using IBM SPSS Statistics 25 (IBM Corp., Armonk, NY, USA).

\section{Device Description and Implantation Technique}

Figure 1 depicts the iStent (containing 1 stent) and iStent inject (containing 2 stents) trabecular micro-bypass systems with their respective dimensions. When implanted through the trabecular meshwork into Schlemm's canal, the stents are designed to increase outflow of aqueous humour from the anterior chamber and thereby decrease IOP. The implantation procedure for each device has been detailed previously. ${ }^{5,19}$ In brief, the surgeon first places the injector through the existing phacoemulsification incision (or makes a new smaller peripheral clear corneal incision if stent implantation is completed as a standalone procedure). Next, the injector is advanced across the anterior chamber to the nasal angle, where the stent is placed into the trabecular meshwork (using a slightly angled approach for iStent or a flush straightforward approach for iStent inject). Then, in the case of an iStent inject implantation, the injector is repositioned within the anterior chamber (without exiting the eye) and a second stent is implanted approximately two clock hours from the first. At the close of either procedure, the stent position and stability are confirmed via intraoperative gonioscopy, the viscoelastic is removed, and the wound patency is ensured. Following surgery, patients were prescribed a topical antibiotic (Moxifloxacin, 4 times per day for one week) and a topical anti-inflammatory (Dexamethasone, 4 times per day and tapering over four weeks).
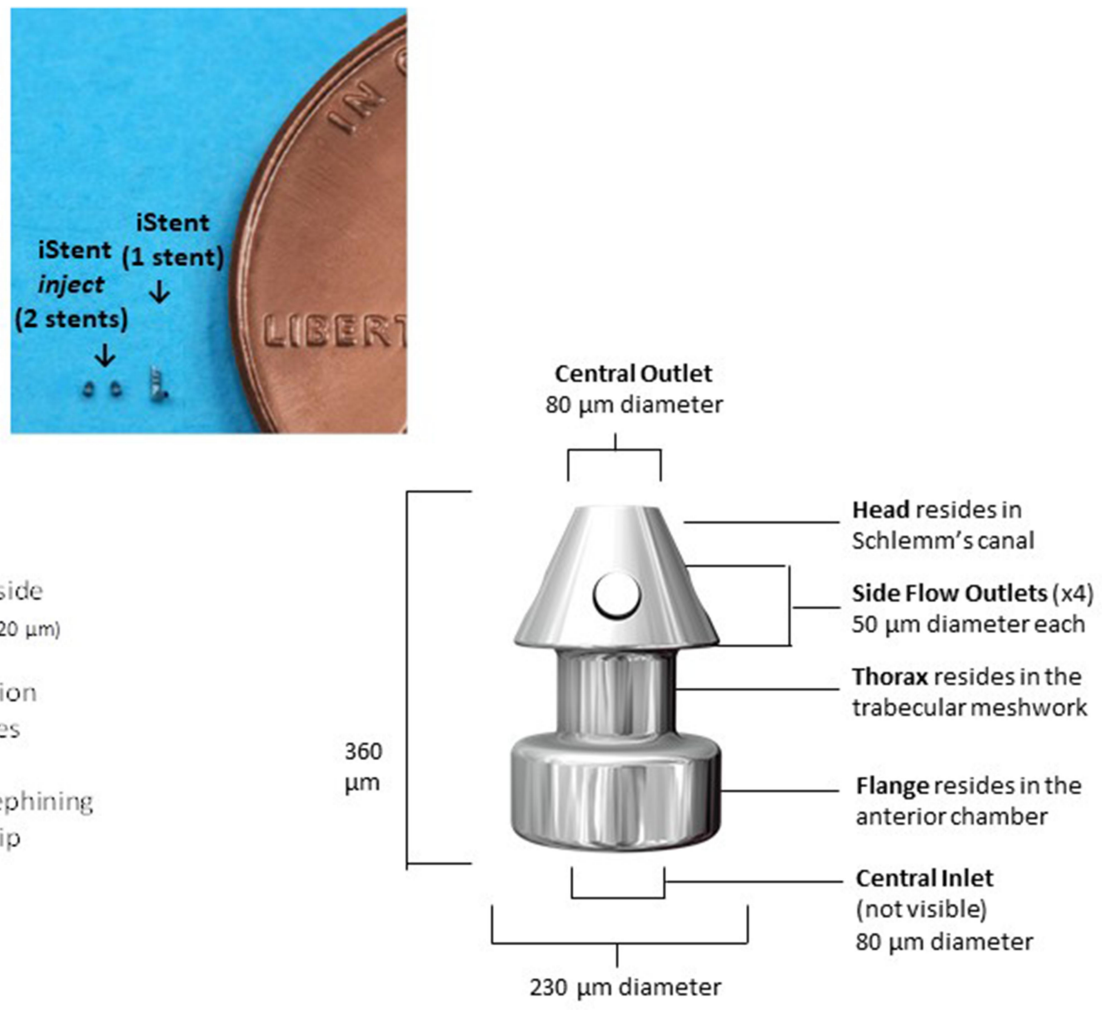

iStent

iStent inject

Figure I iStent ${ }^{\circledR}$ (left) and iStent inject ${ }^{\circledR}$ (right) trabecular micro-bypass stent systems. Image courtesy of Glaukos Corporation (229 Avenida Fabricante, San Clemente, CA 92672; copyright permission March I, 2021). 


\section{Results}

A total of 82 eyes from 82 patients satisfied the inclusion and exclusion criteria and were included in this analysis. Of note, although the present study and the prior two reports ${ }^{38,39}$ were drawn from the same patient base, each manuscript has analyzed a consistent set of patients reaching 6,12 , or 24 months of follow-up by the time of manuscript drafting, respectively, and thus their baseline demographics and postoperative outcomes are not identical.

In the present 24-month consistent cohort, baseline mean \pm standard deviation (SD) age was $70.1 \pm 9.5$ years (range 42-87), with the majority of patients being female (70.27\%) and Caucasian (82.9\%). Glaucoma severity was mild in $79.3 \%$ and moderate in $20.7 \%$ of eyes, according to the Hodapp-Parrish-Anderson visual field criteria (mild, MD no worse than $-6 \mathrm{~dB}$; moderate, MD worse than -6 $\mathrm{dB}$ but no worse than $-12 \mathrm{~dB}$; severe, MD worse than -12 dB). ${ }^{41}$ Approximately three-fourths (74.4\%) of eyes had a diagnosis of primary open-angle glaucoma (POAG), with the remainder consisting of pseudoexfoliation glaucoma $(\mathrm{PXG})$ and pigmentary glaucoma $(\mathrm{PG})$.

Out of the study population, the iStent group comprised 39 eyes $(47.6 \%)$ and the iStent inject group comprised 43 eyes (52.4\%). Table 1 presents the comparison between groups. The groups were well-balanced according to baseline IOP, race, laterality (right or left eye), type of glaucoma, glaucoma stage, and baseline visual acuity. The groups were statistically different according to mean age (the iStent inject group was an average of 9 years older; not independently clinically significant); gender (the iStent group had a higher proportion of female patients; not independently clinically significant); and baseline number of medications (2.2 mean medications in iStent inject eyes and 1.7 medications in iStent eyes).

\section{IOP Results}

Prior to surgery, the mean IOP was higher (although not significantly) in iStent inject eyes than in iStent eyes (17.7 $\mathrm{mmHg}$ vs $16.4 \mathrm{mmHg}$, respectively; $\mathrm{p}=0.147$ ). However, by 12 months postoperative, the mean IOP was lower, and the percent reduction was greater, in iStent inject eyes (26.0\% reduction, from $17.7 \mathrm{mmHg}$ to $13.1 \mathrm{mmHg}$ ) than in iStent eyes (11.0\% reduction, from $16.4 \mathrm{mmHg}$ to 14.8 $\mathrm{mmHg}$ ) (between-groups comparison of percent reduction, $\mathrm{p}=0.002$ ). This between-group difference persisted through

Table I Baseline Characteristics for Each Group

\begin{tabular}{|c|c|c|c|c|}
\hline \multicolumn{2}{|l|}{ Characteristics } & $\begin{array}{c}\text { iStent + Cataract Surgery } \\
n=39\end{array}$ & $\begin{array}{c}\text { iStent Inject + Cataract Surgery } \\
n=43\end{array}$ & p-value \\
\hline \multicolumn{2}{|c|}{ Age (mean $\pm S D)$, years } & $65.7 \pm 9.3$ & $74.1 \pm 7.8$ & $<0.001^{\mathrm{a}}$ \\
\hline \multicolumn{2}{|l|}{ Baseline IOP, $\mathrm{mmHg}$} & $16.4 \pm 3.8$ & $17.7 \pm 4.0$ & $0.147^{\mathrm{a}}$ \\
\hline \multicolumn{2}{|c|}{ Baseline number of medications } & $1.7 \pm 0.8$ & $2.2 \pm 1.0$ & $0.035^{\mathrm{a}}$ \\
\hline Race & $\begin{array}{l}\text { Caucasian } \\
\text { African descent or mixed descent }\end{array}$ & $\begin{array}{l}74.4 \% \\
25.6 \%\end{array}$ & $\begin{array}{c}90.7 \% \\
9.3 \%\end{array}$ & $0.058^{\mathrm{b}}$ \\
\hline Sex & $\begin{array}{l}\text { Male } \\
\text { Female }\end{array}$ & $\begin{array}{l}17.9 \% \\
82.1 \%\end{array}$ & $\begin{array}{l}39.5 \% \\
60.5 \%\end{array}$ & $0.032^{\mathrm{b}}$ \\
\hline Glaucoma Stage & $\begin{array}{l}\text { Early } \\
\text { Moderate }\end{array}$ & $\begin{array}{l}84.6 \% \\
15.4 \%\end{array}$ & $\begin{array}{l}74.4 \% \\
25.6 \%\end{array}$ & $0.255^{\mathrm{b}}$ \\
\hline Laterality & $\begin{array}{l}\text { OD } \\
\text { OS }\end{array}$ & $\begin{array}{l}51.3 \% \\
48.7 \%\end{array}$ & $\begin{array}{l}51.2 \% \\
48.8 \%\end{array}$ & $0.991^{b}$ \\
\hline Type of Glaucoma & $\begin{array}{l}\text { POAG } \\
\text { Other OAG }\end{array}$ & $\begin{array}{l}64.1 \% \\
35.9 \%\end{array}$ & $\begin{array}{l}83.7 \% \\
16.3 \%\end{array}$ & $0.057^{b}$ \\
\hline Baseline visual acuity & $\begin{array}{l}20 / 30 \text { or better } \\
20 / 40 \text { to } 20 / 100 \\
20 / 200 \text { or worse }\end{array}$ & $\begin{array}{c}53.8 \% \\
38.5 \% \\
7.7 \%\end{array}$ & $\begin{array}{c}62.8 \% \\
32.6 \% \\
4.7 \%\end{array}$ & $0.673^{b}$ \\
\hline
\end{tabular}

Notes: ${ }^{\text {a}}$ Student $T$ test; ${ }^{\mathrm{b}} \mathrm{Chi}$-square test.

Abbreviations: SD, standard deviation; IOP, intraocular pressure; OD, right eye; OS, left eye, POAG, primary open-angle glaucoma; OAG, open-angle glaucoma. 
24 months postoperative, when iStent inject eyes continued to display a $26.0 \%$ IOP reduction (from $17.7 \mathrm{mmHg}$ to $13.1 \mathrm{mmHg}$ ) versus a $9.8 \%$ reduction in iStent eyes $(16.4$ $\mathrm{mmHg}$ to $14.8 \mathrm{mmHg}$ ) (between-groups comparison of percent reduction, $\mathrm{p}=0.019)$. Figure 2 depicts mean IOP throughout the 24-month follow-up period for each study group. Table 2 provides the complete listing of mean IOP values for each time point, including statistical comparisons between and within groups. Reduction in IOP values from baseline achieved statistical significance at all time points (from Day 1 to Month 24) for both groups, with the exception of Day 15 in the iStent group. Figure 3 specifically highlights analyses at the 12-month and 24month time points versus baseline.

Additional analyses were completed with stratification by baseline IOP: 40 eyes with baseline IOP $\leq 16 \mathrm{mmHg}$ (23 iStent, 17 iStent inject); 17 eyes with baseline IOP 17-18 $\mathrm{mmHg}$ (6 iStent, 11 iStent inject); and 25 eyes with baseline IOP $\geq 19 \mathrm{mmHg}$ (10 iStent, 15 iStent inject) (Chi-Square: $\mathrm{p}=0.204)$. Within both the iStent and iStent inject groups, postoperative IOP reduction was significantly greater in eyes with higher baseline IOP ( $<<0.001$ in each group) [Figure 3].

Table 3 shows the rates of achieving different levels of target postoperative IOP for each group, either by allowing for medication usage ("qualified success") or with no medications ("unqualified success"). At 24 months after surgery, both groups had higher proportions of eyes achieving the success criteria compared to baseline; the increases reached statistical significance for most cutoffs in iStent eyes, and for all cutoffs in iStent inject eyes.

A survival analysis also was conducted using KaplanMeier survival curves (Figure 4A and B). Mean survival time for an IOP $<18 \mathrm{mmHg}$ with or without medications was 25.4 months (95\% CI: 20.6-30.2) for the iStent group and 25.3 months (95\% CI: 22.4-28.3) for the iStent inject group. Mean survival time for an IOP $<18 \mathrm{mmHg}$ with no medications was 21.9 months (95\% CI: 16.8-27.0) for the iStent group and 21.4 months (95\% CI: 17.7-25.2) for the iStent inject group.

Probability of success for an IOP $<18 \mathrm{mmHg}$ with or without medications was $66.7 \%$ and $81.4 \%(p=0.126$, Log Rank, Mantel-Cox) for the iStent and iStent inject groups, respectively. Probability of success for an IOP $<18 \mathrm{mmHg}$ with no medications was $56.4 \%$ and $67.4 \%(p=0.305$, Log Rank, Mantel-Cox) for the iStent and iStent inject groups, respectively.

Although sample size was limited, an exploratory subgroup analysis was conducted of eyes with secondary

\section{IOP evolution}

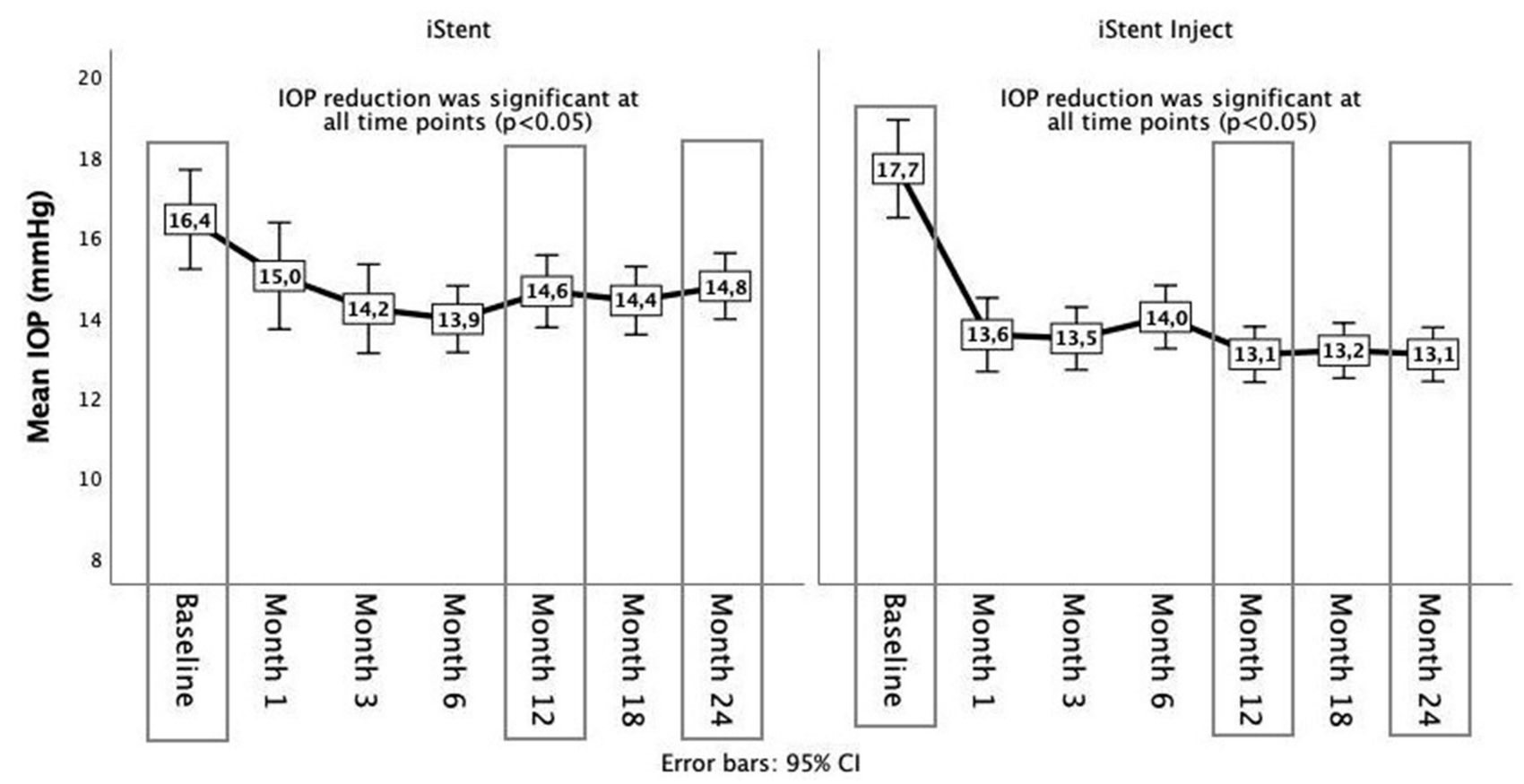

Figure 2 Mean IOP from baseline through 24 months in each study group. 
Table 2 Mean Intraocular Pressure Through 24 Months, with Comparisons Between and Within Study Groups

\begin{tabular}{|c|c|c|c|}
\hline Time Point & $\begin{array}{c}\text { iStent }+ \text { Cataract Surgery } \\
n=39\end{array}$ & $\begin{array}{c}\text { iStent Inject + Cataract Surgery } \\
n=43\end{array}$ & Comparisons Between Groups \\
\hline Baseline IOP & $16.4 \mathrm{mmHg}$ & $17.7 \mathrm{mmHg}$ & 0.147 \\
\hline Day I & $14.2 \mathrm{mmHg}$ & $12.7 \mathrm{mmHg}$ & 0.107 \\
\hline Comparison vs Baseline $^{a}$ & 0.001 & $<0.001$ & \\
\hline Day I5 & $15.5 \mathrm{mmHg}$ & $14.7 \mathrm{mmHg}$ & 0.370 \\
\hline Comparison vs Baseline $^{a}$ & 0.211 & $<0.001$ & \\
\hline Month I & $15.0 \mathrm{mmHg}$ & $13.6 \mathrm{mmHg}$ & 0.066 \\
\hline Comparison vs Baseline $^{a}$ & 0.040 & $<0.001$ & \\
\hline Month 3 & $14.2 \mathrm{mmHg}$ & $13.5 \mathrm{mmHg}$ & 0.266 \\
\hline Comparison vs Baseline $^{a}$ & 0.002 & $<0.001$ & \\
\hline Month 6 & $13.9 \mathrm{mmHg}$ & $14.0 \mathrm{mmHg}$ & 0.928 \\
\hline Comparison vs Baseline $^{a}$ & 0.001 & $<0.001$ & \\
\hline Month 9 & $14.6 \mathrm{mmHg}$ & $13.6 \mathrm{mmHg}$ & 0.204 \\
\hline Comparison vs Baseline $^{a}$ & 0.033 & $<0.001$ & \\
\hline Month 12 & $14.6 \mathrm{mmHg}$ & $13.1 \mathrm{mmHg}$ & 0.006 \\
\hline Comparison vs Baseline $^{a}$ & 0.003 & $<0.001$ & \\
\hline Month 18 & $14.4 \mathrm{mmHg}$ & $13.2 \mathrm{mmHg}$ & 0.023 \\
\hline Comparison vs Baseline $^{a}$ & 0.004 & $<0.001$ & \\
\hline Month 24 & $14.8 \mathrm{mmHg}$ & $13.1 \mathrm{mmHg}$ & 0.002 \\
\hline Comparison vs Baseline $^{a}$ & 0.015 & $<0.001$ & \\
\hline
\end{tabular}

Notes: Bold text indicates key data points (Baseline, Month 12, and Month 24); ${ }^{a}$ Student paired $t$-test, ${ }^{b}$ Student two-sample $t$-test.

Abbreviation: IOP, intraocular pressure.

glaucoma (PXG or PG, $\mathrm{n}=21$ ) that underwent implantation of either stent model. At 24 months following surgery, mean IOP in this subgroup reduced by $22.8 \%$ versus baseline, from $18.4 \mathrm{mmHg}$ to $14.2 \mathrm{mmHg}(\mathrm{p}=0.001)$. This was generally consistent with the outcomes seen in the POAG subgroup $(\mathrm{n}=61)$, which experienced an $18.1 \%$ IOP reduction versus baseline, from $16.6 \mathrm{mmHg}$ to $13.6 \mathrm{mmHg}$ $(\mathrm{p}<0.001)$.

\section{Reduction in Medications}

The reduction in mean number of medications versus baseline achieved statistical significance at all time points ( $<<0.001)$ during 24-month follow-up for both groups (Figure 5 and Table 4). At both Month 12 and Month 24, the groups achieved similarly significant reductions in mean number of medications versus baseline. The iStent group experienced a $73.6 \%$ medication reduction at 12 months (from 1.74 to 0.46 mean medications, $\mathrm{p}<0.001$ ), and a $70.7 \%$ reduction at 24 months (from 1.74 to 0.51 mean medications, $\mathrm{p}<0.001$ ); the iStent inject group experienced a $70.3 \%$ medication reduction at 12 months (from 2.19 to 0.65 mean medications, $\mathrm{p}<0.001$ ) as well as at 24 months. These reductions vs. baseline were statistically similar in the two groups at both time points $(\mathrm{p}=0.841$ and $\mathrm{p}=0.748$ for between-groups comparison of percent reduction at the 12 and 24-month time points, respectively).

Figure $6 \mathrm{~A}-\mathrm{B}$ shows the distribution of number of medications at baseline and at 24 months. Preoperatively, 59.0\% of iStent eyes and $69.8 \%$ of iStent inject eyes were on 2 or more glaucoma medications; by 24 months, this had decreased to $17.9 \%$ of iStent eyes and $20.9 \%$ of iStent inject eyes. At 24 months, $71.8 \%$ of iStent eyes and $65.1 \%$ of iStent 


\section{Mean IOP reduction $(\mathrm{mmHg})$ per level of baseline IOP}

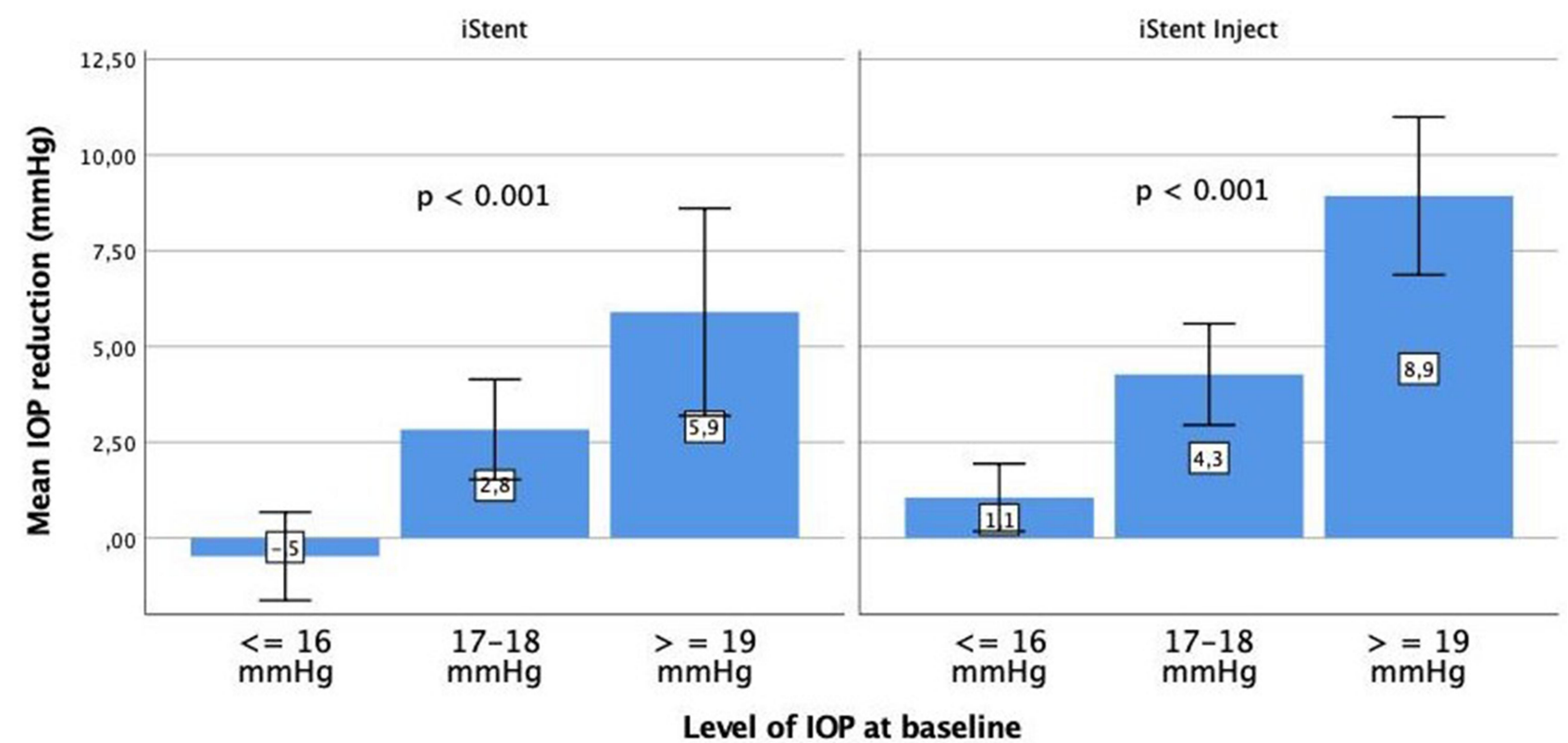

Error bars: + /- $2 \mathrm{SE}$

Figure 3 Mean IOP reduction $(\mathrm{mmHg})$ according to different levels of baseline IOP.

inject eyes were medication-free $(\mathrm{p}=0.832$ for betweengroups comparison), versus $5.1 \%$ of iStent eyes and $2.3 \%$ of iStent inject eyes at baseline.

Within the subgroup analysis of secondary glaucoma cases (PXG and PG, $\mathrm{n}=21$ ), mean number of medications at 24 months reduced by $51.3 \%$ versus baseline, from 1.95 to 0.95 mean medications $(\mathrm{p}<0.001)$. In the POAG subgroup $(\mathrm{n}=61)$, the mean number of medications reduced by $75.8 \%$, from 1.98 to $0.48(\mathrm{p}<0.001)$.

\section{Visual Acuity Results}

There was a significant improvement in the visual acuity in both groups after phacoemulsification-stent surgery. While $53.8 \%$ of iStent eyes and $62.8 \%$ of iStent inject eyes (between-groups comparison $\mathrm{p}=0.673$ ) had a visual acuity of $20 / 30$ or better at baseline, $82.1 \%$ of iStent eyes and $93.0 \%$ of iStent inject eyes (between-groups comparison $\mathrm{p}=0.119$ ) attained this visual acuity level at 24 months. Within-group comparisons of 24-month visual

Table 3 Qualified and Unqualified Success Rates for Different IOP Targets

\begin{tabular}{|c|c|c|c|c|c|c|c|c|}
\hline \multirow{2}{*}{\multicolumn{2}{|c|}{ Success Criteria }} & \multicolumn{3}{|c|}{$\begin{array}{c}\text { iStent }+ \text { Cataract Surgery } \\
n=39\end{array}$} & \multicolumn{3}{|c|}{$\begin{array}{c}\text { iStent Inject }+ \text { Cataract Surgery } \\
n=43\end{array}$} & \multirow{2}{*}{$\begin{array}{c}\text { Between- } \\
\text { Group } \\
\text { Comparison at } \\
\text { M24 }\end{array}$} \\
\hline & & Baseline & $\begin{array}{c}\text { Month } \\
24\end{array}$ & $\begin{array}{l}\text { Within-Group } \\
\text { Comparison } \\
\text { (Baseline vs } \\
\text { M24) }\end{array}$ & Baseline & $\begin{array}{c}\text { Month } \\
24\end{array}$ & $\begin{array}{l}\text { Within-Group } \\
\text { Comparison } \\
\text { (Baseline vs } \\
\text { M24) }\end{array}$ & \\
\hline \multirow[t]{2}{*}{$1 O P<18 \mathrm{mmHg}$} & Qualified & $71.8 \%$ & $89.7 \%$ & 0.092 & $48.8 \%$ & $97.7 \%$ & $<0.001$ & 0.151 \\
\hline & Unqualified & $2.6 \%$ & $66.7 \%$ & $<0.001$ & $0.0 \%$ & $65.1 \%$ & $<0.001$ & 0.534 \\
\hline \multirow[t]{2}{*}{ IOP $<15 \mathrm{mmHg}$} & Qualified & $33.3 \%$ & $82.1 \%$ & $<0.001$ & $20.9 \%$ & $90.7 \%$ & $<0.001$ & 0.206 \\
\hline & Unqualified & $2.6 \%$ & $66.7 \%$ & $<0.001$ & $0.0 \%$ & $60.5 \%$ & $<0.001$ & 0.363 \\
\hline \multirow[t]{2}{*}{$1 O P<12 \mathrm{mmHg}$} & Qualified & $7.7 \%$ & $12.8 \%$ & 0.727 & $2.3 \%$ & $37.2 \%$ & $<0.001$ & 0.011 \\
\hline & Unqualified & $2.6 \%$ & $2.6 \%$ & 1.000 & $0.0 \%$ & $14.0 \%$ & $<0.001$ & 0.071 \\
\hline
\end{tabular}



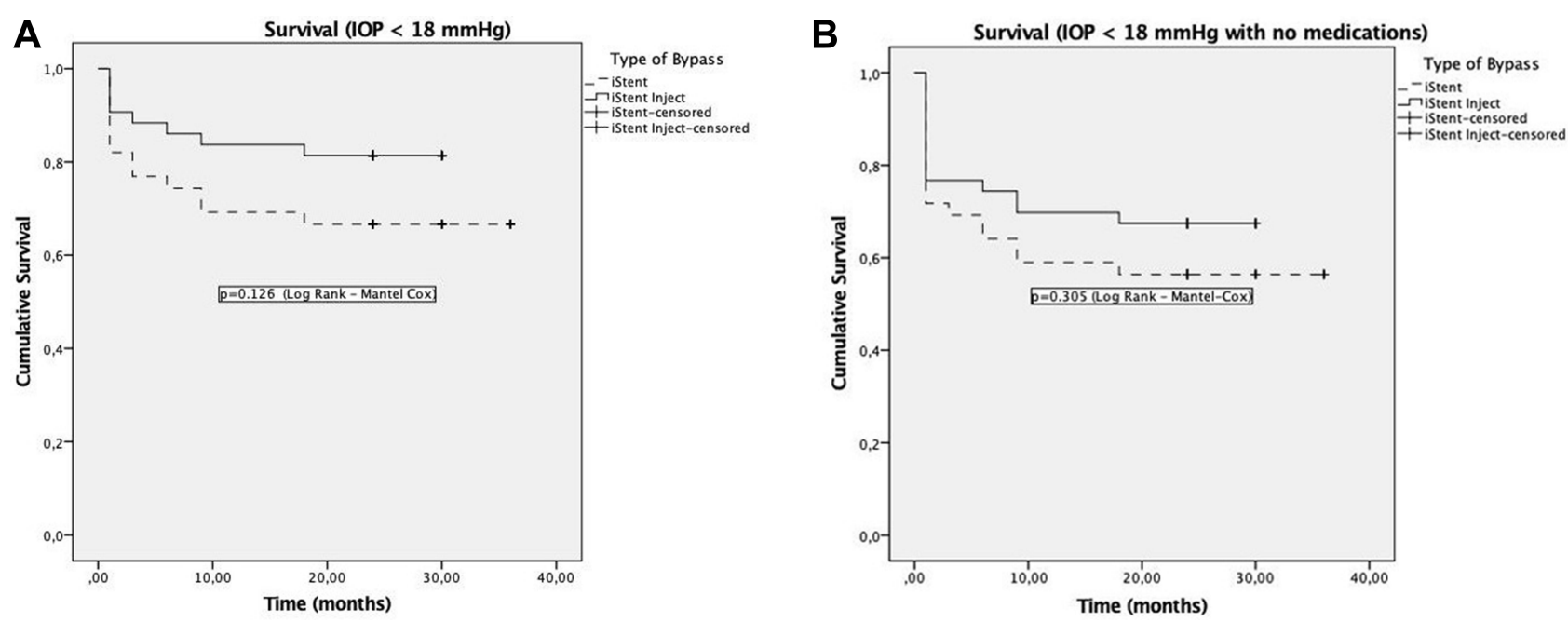

Figure 4 (A) Kaplan-Meier survival analysis for both groups for an IOP $<18 \mathrm{mmHg}$ with or without medications. (B) Kaplan-Meier survival analysis for both groups for an IOP $<18 \mathrm{mmHg}$ with no medications.

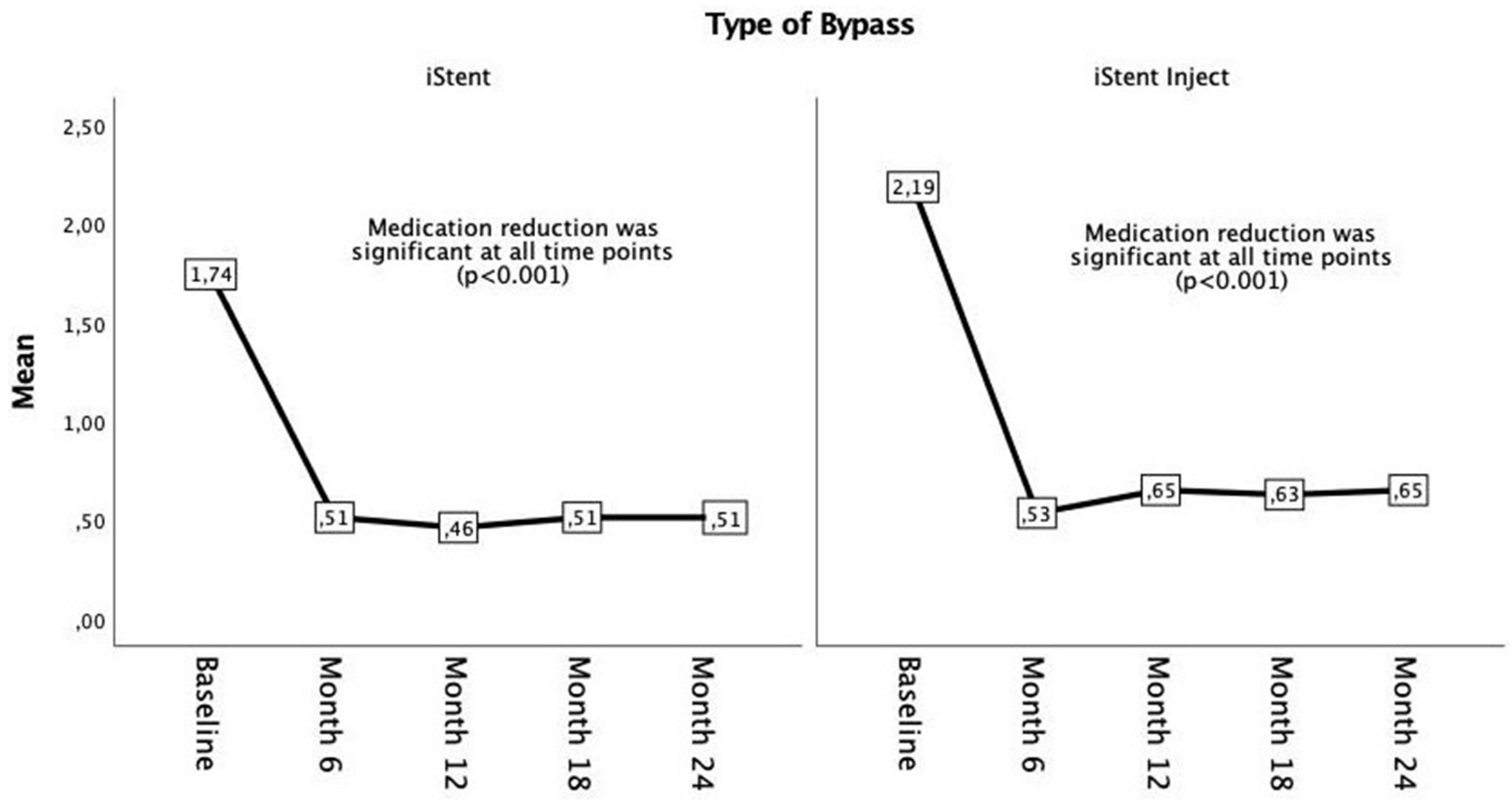

Figure 5 Mean number of medications from baseline to 24 months in each group.

acuity proportions versus baseline were significant for both groups $(\mathrm{p}=0.018$ for iStent and $\mathrm{p}=0.012$ for iStent inject).

\section{Safety Results}

Both groups demonstrated a high safety profile. Intraoperative complications were rare, occurring in 4 eyes in the iStent group (10.3\%) and no eyes in the
iStent inject group (between-groups comparison $\mathrm{p}=0.098$ ). The 4 iStent complications included three cases of excessive blood reflux and one case of stent malpositioning (under- or over-implanted, as identified by gonioscopic examination).

Postoperative adverse events also were infrequent, occurring in 4 eyes (10.3\%) in the iStent group and 1 eye $(2.3 \%)$ in the iStent inject group. In the iStent group, one eye had focal 
Table 4 Mean Number of Medications Through 24 Months, with Comparisons Between and Within Study Groups

\begin{tabular}{|c|c|c|c|}
\hline \multirow[t]{2}{*}{ Time Point } & \multicolumn{2}{|c|}{ Number of Medications per Eye } & \multirow{2}{*}{$\begin{array}{c}\text { Comparisons Between } \\
\text { Groups }^{\text {b }}\end{array}$} \\
\hline & $\begin{array}{c}\text { iStent }+ \text { Cataract } \\
\text { Surgery } \\
n=39\end{array}$ & $\begin{array}{c}\text { iStent Inject + Cataract } \\
\text { Surgery } \\
n=43\end{array}$ & \\
\hline BASELINE & 1.74 & 2.19 & 0.035 \\
\hline Day I & 0.10 & 0.14 & 0.731 \\
\hline Comparison vs Baseline $^{a}$ & $<0.001$ & $<0.001$ & \\
\hline Day 15 & 0.26 & 0.33 & 0.670 \\
\hline Comparison vs Baseline $^{a}$ & $<0.001$ & $<0.001$ & \\
\hline Month I & 0.44 & 0.40 & 0.817 \\
\hline Comparison vs Baseline $^{\mathrm{a}}$ & $<0.001$ & $<0.001$ & \\
\hline Month 3 & 0.46 & 0.51 & 0.804 \\
\hline Comparison vs Baseline $^{\mathrm{a}}$ & $<0.001$ & $<0.001$ & \\
\hline Month 6 & 0.51 & 0.53 & 0.913 \\
\hline Comparison vs Baseline $^{a}$ & $<0.001$ & $<0.001$ & \\
\hline Month 9 & 0.51 & 0.63 & 0.599 \\
\hline Comparison vs Baseline $^{a}$ & $<0.001$ & $<0.001$ & \\
\hline MONTH I 2 & 0.46 & 0.65 & 0.398 \\
\hline Comparison vs Baseline $^{a}$ & $<0.001$ & $<0.001$ & \\
\hline Month 18 & 0.51 & 0.63 & 0.594 \\
\hline Comparison vs Baseline $^{a}$ & $<0.001$ & $<0.001$ & \\
\hline MONTH 24 & 0.51 & 0.65 & 0.521 \\
\hline Comparison vs Baseline $^{a}$ & $<0.001$ & $<0.001$ & \\
\hline
\end{tabular}

Notes: Bold text indicates key data points (Baseline, Month 12, and Month 24). ${ }^{a}$ Student paired $t$-test, ${ }^{\mathrm{b}}$ Student two-sample $t$-test. Abbreviation: IOP, intraocular pressure.

peripheral anterior synechia occluding the internal ostium of the iStent, which was corrected by Nd:YAG laser goniosynechialysis with no resultant sequelae. Three other iStent eyes had uncontrolled IOP during follow-up (all of them between 3 and 6 months postoperative) and underwent filtration surgery: in two eyes due to steroid-induced IOP spike from systemic steroid use, which the patients required for pulmonary and rheumatologic diseases; and in one eye due to allergies to all ocular hypotensive agents and the need for more IOP reduction than what was achieved by the stent. In the iStent inject group, one eye had insufficient IOP control and underwent a filtration surgery at 3 months. The IOP and medication values recorded after filtration surgery in these four eyes were excluded from subsequent efficacy and survival curve analyses.

\section{Discussion}

This paper constitutes some of the first comparative twoyear data on the first- and second-generation trabecular micro-bypass stents, iStent and iStent inject. Significant reductions in IOP and medications were observed with either device, along with a favourable safety profile. As with the one-year data, the IOP and medication reductions from baseline were significant in both groups, with greater gains observed after iStent inject than iStent. The degree of postoperative IOP reduction also was greater in eyes with higher preoperative IOP, as has been shown previously. ${ }^{10,12-14,21}$ Importantly, the outcomes in both groups were achieved in consecutive (all-comer) patients in the context of the surgeon's clinical practice, with the patient population including different glaucoma subtypes 

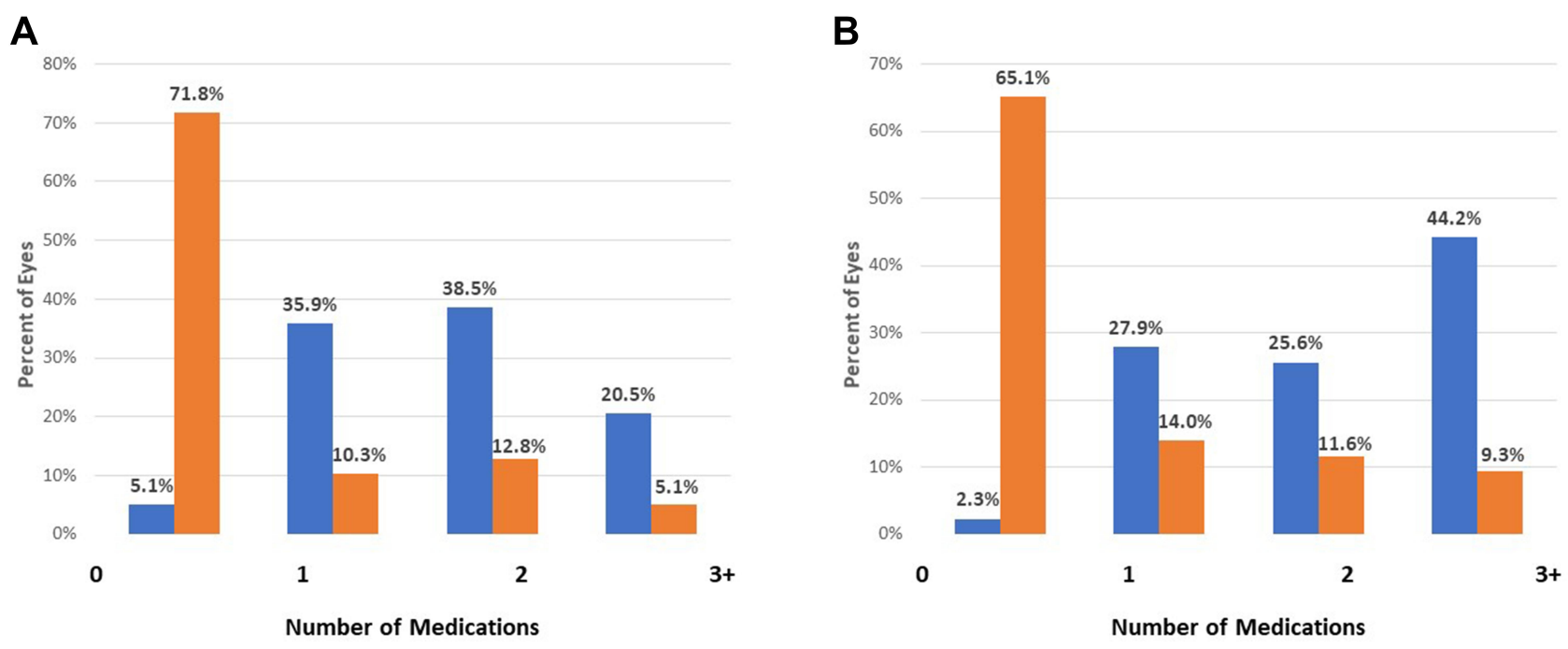

Figure 6 Distribution of number of medications at baseline and at 24 months, iStent group (A) and iStent inject group (B).

and severities. The subgroup analysis of secondary glaucoma cases (PXG and PG), although limited in size, revealed favourable IOP and medication reductions, consistent with previously published larger studies of the iStent specifically in these populations. ${ }^{12,13}$ Altogether, these study attributes reinforce the veracity and applicability of the data to the diverse real-world settings in which surgeons and patients make treatment decisions.

The IOP reduction following iStent inject was consistently greater than that of iStent. This between-group difference was apparent at 12 months and remained present to a similar or greater degree at 24 months. Higher proportions of iStent inject eyes than iStent eyes also met the prespecified effectiveness endpoints (IOP $<18,15,12$ $\mathrm{mmHg}$ ) at 24 months. These between-group differences have meaningful consequences given that every $1 \mathrm{mmHg}$ of IOP reduction has been shown to equate to a $10 \%$ lower risk of glaucoma development ${ }^{42}$ and a $10-19 \%$ lower risk of glaucoma progression. ${ }^{43,44}$ Alongside the reductions in IOP, both groups experienced significant reductions in medication burden from baseline. This was manifested not only in a decreased mean medication burden (from 1.74 to 0.51 medications in iStent eyes, and from 2.19 to 0.65 medications in iStent inject eyes), but also in a threefold decrease in the proportion of eyes on $\geq 2$ medications, and a 14- to 28 -fold increase in the percentage of eyes becoming eyedrop-free versus baseline.

The benefits of medication reduction are widely varied and highly impactful. Topical medications are known to degrade the conjunctiva and increase the risk of future surgical failure. ${ }^{45}$ Their effect is limited by relatively high rates of eyedrop non-compliance, which increases the risk of visual loss and disease progression. ${ }^{46}$ Even when perfect compliance is present, the diurnal IOP fluctuations of any non-surgical treatment may place patients at greater risk of disease progression. ${ }^{47}$ Topical medications also are known to promote ocular surface disease (OSD) and dry eye, ${ }^{48}$ and to diminish the quality of life (QoL). ${ }^{49}$

Although the goal of medication reduction is important to all glaucoma patients, it is especially germane to patients undergoing MIGS procedures. MIGS patients typically have mild to moderate disease severity that does not necessitate the dramatic IOP reductions of traditional filtration surgeries, nor warrant the risks associated with such surgeries. In many of these patients, the primary goal of stent implantation may be medication reduction with its associated OSD and QoL benefits (provided that IOP stay at a similar or lower level than preoperative).

The impact of MIGS surgery on OSD and QoL is an increasingly important area of research. To date, one study has specifically examined the change in OSD, ${ }^{31}$ and one pivotal trial has analyzed the change in QoL, ${ }^{37}$ following MIGS surgery and its attendant medication reductions; both studies were completed in eyes undergoing iStent or iStent inject implantation. The OSD analysis by Schweitzer et al showed significant improvements in objective examination findings and subjective symptom questionnaires following iStent or iStent inject implantation. ${ }^{31}$ The first QoL analysis within a MIGS pivotal trial, completed within the iStent inject trial, showed significantly higher rates of QoL and OSD 
improvement in stent eyes than in control eyes. ${ }^{37}$ In light of the findings of these two studies, the substantial medication reduction observed in the present study is particularly important and relevant.

There were certain limitations in the current 24-month analysis. The data were collected retrospectively and were drawn from a single surgeon and site. Preoperative mean IOP was slightly lower in the iStent group compared to the iStent inject group; although the difference was not statistically significant, it could have mildly influenced the percentage IOP reduction that could be reached postoperatively (given the previously discussed correlation between higher preoperative IOP and greater postoperative percentage reduction). Stent implantation was completed with concomitant cataract surgery, so the stent effect could not be isolated from the effect of cataract surgery. However, since each group would be affected by phacoemulsification equally, the between-group differences are still meaningful; and within each group, the preoperative measures of the patients could serve as their own control, so any preoperative-versus-postoperative comparisons remain relevant. Finally, the subgroup analysis of secondary glaucoma cases showed favourable preliminary trends, consistent with prior research, ${ }^{12,13}$ but further studies will be important to more fully assess stent performance in these subtypes.

\section{Conclusions}

In summary, this real-world retrospective analysis provides novel two-year information on the comparative safety and effectiveness of the first-generation iStent and second-generation iStent inject trabecular microbypass stents. Through 24 months postoperative, both devices produced sustained and significant reductions in IOP and medication burden, while maintaining favourable safety. Comparatively, the iStent inject group experienced consistently greater IOP reductions and higher proportions of eyes meeting the prespecified IOP endpoints than the iStent group. Within both groups, higher preoperative IOP was associated with greater postoperative IOP reductions. Mean medication reduction was similarly substantial in both groups (approximately 70\% decrease from preoperative), with both groups also exhibiting a dramatic increase in the percent of eyes being medication-free.

\section{Compliance with Ethics Guidelines}

The study was performed in accordance with the ethical standards of the Declaration of Helsinki (as revised in
Brazil 2013), including informed consent of patients; it was approved by the Ethics Committee of the Santa Casa de Misericordia de Juiz de Fora.

\section{Authorship Verification}

All authors contributed to data analysis, drafting or revising the article, have agreed on the journal to which the article will be submitted, gave final approval of the version to be published, and agree to be accountable for all aspects of the work.

\section{Funding}

No financial support was received for the work in this study. Article processing fees and editorial assistance were provided by Glaukos Corporation (San Clemente, California, USA).

\section{Disclosure}

Dr. Ricardo Guedes has the following disclosures: nonfinancial support from Glaukos Corp., consultant to Glaukos, Théa France, OftaVision Brasil, Alcon, Allergan. Dr. Daniela Marcelo Gravina has the following disclosures: none Dr. Vanessa Maria Paletta Guedes has the following disclosures: none Dr. Alfredo Chaoubah has the following disclosures: none.

\section{References}

1. Tham YC, Li X, Wong TY, et al. Global prevalence of glaucoma and projections of glaucoma burden through 2040: a systematic review and meta-analysis. Ophthalmology. 2014;121:2081-2090. doi:10.1016/j. ophtha.2014.05.013

2. Jampel HD, Musch DC, Gillespie BW, Lichter PR, Wright MM, Guire KE, Collaborative Initial Glaucoma Treatment Study Group. Collaborative Initial Glaucoma Treatment Study Group. Perioperative complications of trabeculectomy in the collaborative initial glaucoma treatment study (CIGTS). Am J Ophthalmol. 2005;140(1):16-22. doi:10.1016/j.ajo.2005.02.013

3. Rulli E, Biagioli E, Riva I, et al. Efficacy and safety of trabeculectomy vs nonpenetrating surgical procedures: a systematic review and meta-analysis. JAMA Ophthalmol. 2013;131(12):1573-1582. doi:10.1001/jamaophthalmol.2013.5059

4. Gedde SJ, Herndon LW, Brandt JD, Budenz DL, Feuer WJ, Schiffman JC. Tube Versus Trabeculectomy Study Group. Postoperative complications in the Tube Versus Trabeculectomy (TVT) study during five years of follow-up. Am $J$ Ophthalmol. 2012;153:804-814. doi:10.1016/j.ajo.2011.10.024

5. Samuelson TW, Katz LJ, Wells JM, Duh Y-J, Giamporcaro JE. Randomized evaluation of the trabecular micro-bypass stent with phacoemulsification in patients with glaucoma and cataract. Ophthalmology. 2011;118(3):459-467. doi:10.1016/j.ophtha.20 10.07.007

6. Belovay GW, Naqi A, Chan BJ, Rateb M, Ahmed IIK. Using multiple trabecular micro-bypass stents in cataract patients to treat open-angle glaucoma. J Cataract Refract Surg. 2012;38(11):1911-1917. doi:10.1016/j.jcrs.2012.07.017 
7. Chen DZ, Sng CCA, Sangtam T, et al. Phacoemulsification vs phacoemulsification with micro-bypass stent implantation in primary angle closure and primary angle closure glaucoma: a randomized single-masked clinical study. Clin Exp Ophthalmol. 2020;48 (4):450-461. doi:10.1111/ceo.13721

8. Neuhann TH, Hornbeak DM, Neuhann RT, Giamporcaro JE. Longterm effectiveness and safety of trabecular micro-bypass stent implantation with cataract surgery in patients with glaucoma or ocular hypertension: 5-year outcomes. J Cataract Refract Surg. 2019;45(3):312-320. doi:10.1016/j.jcrs.2018.10.029

9. Gallardo MJ, Supnet RA. Three-year outcomes of combined trabecular micro-bypass and phacoemulsification in a predominantly Hispanic population with primary open-angle glaucoma. Clin Ophthalmol. 2019;13:869-879. doi:10.2147/OPTH.S189071

10. Ferguson TJ, Mechels KB, Dockter Z, et al. iStent trabecular microbypass stent implantation with phacoemulsification in patients with open-angle glaucoma: 6-year outcomes. Clin Ophthalmol. 2020;14:1859-1866. doi:10.2147/OPTH.S247910

11. Ferguson T, Swan R, Ibach M, Schweitzer J, Sudhagoni R, Berdahl JP. Evaluation of a trabecular microbypass stent with cataract extraction in severe primary open-angle glaucoma. J Glaucoma. 2018;27(1):71-76. doi:10.1097/IJG.0000000000000825

12. Ferguson TJ, Swan RJ, Bleeker A, et al. Trabecular microbypass stent implantation in pseudoexfoliative glaucoma: long-term results [published online ahead of print, 2020 May 7]. J Cataract Refract Surg. 2020. doi: $10.1097 /$ j.jcrs. 0000000000000243

13. Ferguson TJ, Ibach M, Schweitzer J, Karpuk KL, Stephens JD, Berdahl JP. Trabecular microbypass stent implantation with cataract extraction in pigmentary glaucoma. Clin Exp Ophthalmol. 2020;48 (1):37-43. doi:10.1111/ceo.13638

14. Ferguson TJ, Ibach M, Schweitzer J, et al. Trabecular microbypass stent implantation in pseudophakic eyes with open-angle glaucoma: long-term results. J Cataract Refract Surg. 2019;45(4):414-420. doi:10.1016/j.jcrs.2018.11.005

15. Nitta K, Yamada Y, Morokado S, Sugiyama K. iStent trabecular micro-bypass stent implantation with cataract surgery in a Japanese glaucoma population. Clin Ophthalmol. 2020;15(14):3381-3391. doi: $10.2147 /$ OPTH.S274281

16. Fechtner RD, Voskanyan L, Vold SD, et al. Five-year, prospective, randomized, multi-surgeon trial of two trabecular bypass stents versus prostaglandin for newly-diagnosed open-angle glaucoma. Ophthalmology Glaucoma. 2019;2(3):156-166. doi:10.1016/j.ogla.2019.03.004

17. Shalaby WS, Jia J, Katz LJ, Lee D. iStent inject ${ }^{\circledR}$ : a comprehensive survey of the literature [published online ahead of print, $2020 \mathrm{Jul} \mathrm{10].}$ J Cataract Refract Surg. 2020. doi:10.1097/j.jcrs.0000000000000325

18. Clement C, Howes F, Ioannidis AS, et al. Two-year multicenter outcomes of iStent inject trabecular micro-bypass stents combined with phacoemulsification in various types of glaucoma and ocular hypertension. Clin Ophthalmol. 2020;14:3507-3517. doi:10.2147/OPTH.S271646

19. Samuelson TW, Sarkisian SR Jr, Lubeck DM, et al. for the iStent inject Study group, prospective, randomized, controlled pivotal trial of iStent inject trabecular micro-bypass in primary open-angle glaucoma and cataract: two-year results. Ophthalmology. 2019;126 (6):811-821. doi:10.1016/j.ophtha.2019.03.006

20. Neuhann R, Neuhann T. Second-generation trabecular micro-bypass stent implantation: retrospective analysis after 12- and 24-month follow-up. Eye Vis (Lond). 2020;7:1. doi:10.1186/s40662-019-0169-7

21. Ferguson TJ, Dockter Z, Bleeker A, et al. iStent inject trabecular microbypass stent implantation with cataract extraction in open-angle glaucoma: early clinical experience. Eye Vis (Lond). 2020;7:28. doi:10.1186/s40662-020-00194-3

22. Hengerer FH, Auffarth GU, Riffel C, Conrad-Hengerer I. Prospective, non-randomized, 36-month study of second-generation trabecular micro-bypass stents with phacoemulsification in various types of glaucoma. Ophthalmol Ther. 2018;7(2):405-415. doi:10.1007/s40123-018-0152-8
23. Hengerer FH, Auffarth GU, Riffel C, Conrad-Hengerer I. Secondgeneration trabecular micro-bypass stents as standalone treatment for glaucoma: a 36-month prospective study. Adv Ther. 2019;36 (7):1606-1617. doi:10.1007/s12325-019-00984-9

24. Fea AM, Belda JI, Rekas M, et al. Prospective unmasked randomized evaluation of the iStent inject versus two ocular hypotensive agents in patients with primary open-angle glaucoma. Clin Ophthalmol. 2014;8:875-882. doi:10.2147/OPTH.S59932

25. Lindstrom R, Sarkisian SR, Lewis R, Hovanesian J, Voskanyan L. Four-year outcomes of two second-generation trabecular micro-bypass stents in patients with open-angle glaucoma on one medication. Clin Ophthalmol. 2020;14:71-80. doi:10.2147/OPTH. S235293

26. Huang AS, Penteado RC, Papoyan V, Voskanyan L, Weinreb RN. Aqueous angiographic outflow improvement after trabecular micro-bypass in glaucoma patients. Ophthalmology Glaucoma. 2019;2:11-21. doi:10.1016/j.ogla.2018.11.010

27. Salimi A, Watt H, Harasymowycz P. Three-year outcomes of second-generation trabecular micro-bypass stents (iStent inject) with phacoemulsification in various glaucoma subtypes and severities. J Glaucoma. 2020.

28. Salimi A, Clement C, Shiu M, Harasymowycz P. Second-generation trabecular micro-bypass (iStent inject) with cataract surgery in eyes with normal-tension glaucoma: one-year outcomes of a multi-centre study [published online ahead of print, $2020 \mathrm{Jul} 1$ 1]. Ophthalmol Ther. 2020;9(3):585-596. doi:10.1007/s40123-020-00266-6

29. Scott RA, Ferguson TJ, Stephens JD, Berdahl JP. Refractive outcomes after trabecular microbypass stent with cataract extraction in open-angle glaucoma. Clin Ophthalmol. 2019;13:1331-1340. doi: $10.2147 /$ OPTH.S206619

30. Ioannidis AS, Toteberg-Harms M, Hamann T, Hodge C. Refractive outcomes after trabecular micro-bypass stents (iStent inject) with cataract extraction in open-angle glaucoma. Clin Ophthalmol. 2020;14:517-524. doi:10.2147/OPTH.S239103

31. Schweitzer JA, Hauser WH, Ibach M, et al. Prospective interventional cohort study of ocular surface disease changes in eyes after trabecular micro-bypass Stent(s) Implantation (iStent or iStent inject) with Phacoemulsification [published online ahead of print, 2020 Aug 13]. Ophthalmol Ther. 2020;9(4):941-953. doi:10.1007/s40123-020-00290-6

32. Al Habash A, Nagshbandi AA. Quality of life after combined cataract and minimally invasive glaucoma surgery in glaucoma patients. Clin Ophthalmol. 2020;14:3049-3056. doi:10.2147/OPTH.S276124

33. Ngan K, Fraser E, Buller S, Buller A. A cost minimisation analysis comparing iStent accompanying cataract surgery and selective laser trabeculoplasty versus topical glaucoma medications in a public healthcare setting in New Zealand. Graefes Arch Clin Exp Ophthalmol. 2018;256(11):2181-2189. doi:10.1007/s00417-018-4104-8

34. Patel V, Ahmed I, Podbielski D, Falvey H, Murray J, Goeree R. Costeffectiveness analysis of standalone trabecular micro-bypass stents in patients with mild-to-moderate open-angle glaucoma in Canada. $J$ Med Econ. 2019;22(4):390-401. doi:10.1080/13696998.20 19.1572013

35. Berdahl JP, Khatana AK, Katz LJ, et al. Cost-comparison of two trabecular micro-bypass stents versus selective laser trabeculoplasty or medications only for intraocular pressure control for patients with open-angle glaucoma. $J$ Med Econ. 2017;20(7):760-766. doi:10.1080/13696998.2017.1327439

36. Wang SY, Singh K, Stein JD, Chang RT. Ocular antihypertensive medication use after iStent implantation concurrent with cataract surgery vs cataract surgery alone in a large US health care claims database. JAMA Ophthalmol. 2018;136(4):428-431. doi:10.1001/ jamaophthalmol.2018.4461

37. Samuelson TS Long-term corneal endothelial safety and patient-reported outcomes of iStent inject trabecular micro-bypass. Presentation at the Annual Meeting of the American Society of Cataract and Refractive Surgeons (Virtual): May 16, 2020. 
38. Guedes RAP, Gravina DM, Lake JC, Guedes VMP, Chaoubah A. Intermediate Results of iStent or iStent inject implantation combined with cataract surgery in a real-world setting: a longitudinal retrospective study. Ophthalmol Ther. 2019;8(1):87-100. doi:10.1007/ s40123-019-0166-x

39. Guedes RAP, Gravina DM, Lake JC, Guedes VMP, Chaoubah A. One-year comparative evaluation of iStent or iStent inject implantation combined with cataract surgery in a single center. Adv Ther. 2019;36(10):2797-2810. doi:10.1007/s12325-019-01067-5

40. Manning D. Real-world case series of iStent or iStent inject trabecular micro-bypass stents combined with cataract surgery. Ophthalmol Ther. 2019;8(4):549-561. doi:10.1007/s40123-01900208-x

41. Hodapp E, Parrish RK II, Anderson DR. Clinical Decisions in Glaucoma. St Louis, MO: The CV Mosby Co; 1993.

42. Gordon MO, Beiser JA, Brandt JD, et al. The Ocular Hypertension Treatment Study: baseline factors that predict the onset of primary open-angle glaucoma. Arch Ophthalmol. 2002;120(6):714-720. doi:10.1001/archopht.120.6.714

43. Leske MC, Heijl A, Hussein M, Bengtsson B, Hyman L, Komaroff E. Factors for glaucoma progression and the effect of treatment: the Early Manifest Glaucoma Trial. Arch Ophthalmol. 2003;121 (1):48-56. doi:10.1001/archopht.121.1.48
44. Chauhan BC, Mikelberg FS, Balaszi AG, et al. Canadian Glaucoma Study: 2. risk factors for the progression of open-angle glaucoma. Arch Ophthalmol. 2008;126:1030-1036. doi:10.1001/ archopht.126.8.1030

45. Johnson DH, Yoshikawa K, Brubaker RF, et al. The effect of long-term medical therapy on the outcome of filtration surgery. Am $J$ Ophthalmol. 1994;117:139-148. doi:10.1016/S0002-9394(14) 73068-5

46. Rossi GC, Pasinetti GM, Scudeller L, et al. Do adherence rates and glaucomatous visual field progression correlate? Eur J Ophthalmol. 2011;21:410-414. doi:10.5301/EJO.2010.6112

47. Nouri-Mahdavi K, Hoffman D, Coleman AL, et al. Predictive factors for glaucomatous visual field progression in the Advanced Glaucoma Intervention Study. Ophthalmology. 2004;111:1627-1635. doi:10.1016/j.ophtha.2004.02.017

48. Fechtner RD, Godfrey DG, Budenz D, Stewart JA, Stewart WC, Jasek MC. Prevalence of ocular surface complaints in patients with glaucoma using topical intraocular pressure-lowering medications. Cornea. 2010;29(6):618-621. doi:10.1097/ICO.0b013e3181c325b2

49. Skalicky SE, Goldberg I, McCluskey P. Ocular surface disease and quality of life in patients with glaucoma. Am J Ophthalmol. 2012;153:1-9.e2. doi:10.1016/j.ajo.2011.05.033
Clinical Ophthalmology

\section{Publish your work in this journal}

Clinical Ophthalmology is an international, peer-reviewed journal covering all subspecialties within ophthalmology. Key topics include: Optometry; Visual science; Pharmacology and drug therapy in eye diseases; Basic Sciences; Primary and Secondary eye care; Patient Safety and Quality of Care Improvements. This journal is indexed on PubMed
Dovepress

Central and CAS, and is the official journal of The Society of Clinical Ophthalmology (SCO). The manuscript management system is completely online and includes a very quick and fair peer-review system, which is all easy to use. Visit http://www.dovepress.com/ testimonials.php to read real quotes from published authors. 